M. Fakhimi, D. Robertson, and T. Boness, eds.

DOI: https://doi.org/10.36819/SW21.018

\title{
AN EXPLORATORY STUDY ON THE USES OF "WRONG" DISCRETE EVENT SIMULATION MODELS IN PRACTICE
}

\section{Mr. Naoum Tsioptsias}

Loughborough University

Epinal Way, Loughborough LE11 3TU

n.tsioptsias@lboro.ac.uk
Dr. Antuela Tako

Loughborough University

Epinal Way, Loughborough LE11 3TU

a.takou@lboro.ac.uk

\section{Professor Stewart Robinson}

Loughborough University

Epinal Way, Loughborough LE11 3TU

s.l.robinson@lboro.co.uk

\begin{abstract}
It is generally accepted that no model is perfect. Literature has focused mainly on presenting successful simulation studies. This paper examines the extent to which "wrong" models are used in practice and how modellers and clients deal with them. We interview 22 discrete-event simulation modellers and analyse 54 reallife stories to identify possible uses of "wrong" models. We also focus our analysis on the model's level of detail as a possible reason for considering a model wrong, and analyse how this can affect acceptance and use of a "wrong" model. Results indicate that "wrong" models are indeed utilised in practice even if they are not recognised so by all parties involved. Our results show that usefulness and model acceptance do not necessarily concur. The outcomes of this investigation can be further utilised in future studies to show how modellers and clients may interact when encountering a "wrong" model.
\end{abstract}

Keywords: Wrong models, usefulness, discrete-event simulation, uses, model acceptance

\section{INTRODUCTION}

Simulation modelling is central to the practice of Operational Research (OR). Yet, when creating a model, various issues may be encountered. These could lead to an outcome that may not be considered as adequate by clients or even modellers. Instead, the model may be viewed as "wrong". Should such a model be discarded or can we still use it somehow?

Literature has suggested that "wrong" models may still have certain uses for their users. Papers prefer to focus on successful outcomes (Eskinasi and Fokkema, 2006) not usually including feedback from preceding failed attempts. As a result, we know little about the usefulness of "wrong" models because they are not discussed in the existing published work. The need for this sort of research becomes apparent when dealing with cases of "wrong" models in real life. This paper explores the use of "wrong" models based on suggestions found in the literature. We aim to provide a better understanding of "wrong" models by exploring the extent to which they are used in practice. The main contribution of our work lies in that it provides evidence based on real life stories as described by simulation analysts. We also carry out a focused analysis on one specific reason of wrongness, namely the model's level of detail, and consider how it affects acceptance and use. Modellers may utilise ideas from our empirical analysis presented here and find arguments that justify to their clients that it is possible to take advantage even from a model that they may deem "wrong".

Concerning our approach to the definition of "wrong" models and to their uses, a very important note should be made that applies throughout the context. When discussing "wrong" models and their possible acceptance or use here, we refer to any model element that may be deemed as improper by clients or modellers (or both). We do not equate acceptance or use of a "wrong" model to a modeller 
willingly developing a bad model for their clients. For instance modellers may have to accept a "wrong" model due to their clients' preference, or on the other hand, a perfectly acceptable model by the modeller, may be rejected by the client. Thus wrongness is subjective and "wrong" models may be present even if they are not considered or actually be wrong. To demonstrate that this is our definition to wrongness, we use quotes when referring to opinions on a model considered as "wrong".

To achieve our aims, we interview discrete-event simulation modellers on their experience of using "wrong" models and carry out qualitative text analysis. We focus on how modellers deal with "wrong" models and identify the reasons for using them or not by presenting actual cases of "wrong" models from practice. We first review the literature by exploring model usefulness and possible uses of "wrong" models, also focusing on the model's level of detail and its effect on credibility. Then, the methodology and objectives of the study are provided. Results follow the interview analysis with some first deductions. A summary and discussion of proposed future work conclude the paper.

\section{2 "WRONG" MODELS IN LITERATURE}

In this section we discuss existing views on "wrong" models in literature in terms of their usefulness and possible uses. Some additional concepts relevant to the needs of this work are also analysed.

Considering how "all models are wrong" (Box and Draper, 1987), the concept of "wrongness" is highly diverse in literature. Tsioptsias et al. (2018) reference such examples from various works where the terms include: "bad" or "inadequate" (Hodges, 1991), "unvalidated" or "unvalidatable" (Hodges and Dewar, 1992), "false" or "incorrect" (Bankes, 1998), or simply "wrong" (e.g. Bankes, 1993). Regarding reasons of wrongness, various ideas are found on why models may be considered "wrong", like limitations in time or funding (Balci et al., 2002), decisions (Vennix et al., 1999), etc.

Accordingly, different factors during the modelling study may affect model development. These include the gathering of data and creation of a conceptual model, coding, experimentation, and, implementation of the model (Robinson, 2014). The experimentation or testing phase also contains the Validation and Verification (V\&V) process of the model (Robinson, 2014). V\&V has long occupied literature in terms of different approaches and tests to craft a "proper" model. Their analysis is not within the scope of this paper, yet this has been summarised in Tsioptsias et al. (2016). Lastly, validation relies on "trade-offs" within different validity types and cannot be universal or absolute (Groesser \& Schwaninger, 2012; Sargent, 2012). Thus, we may consider that instead of searching for a "perfect" model, we should be selecting among the available alternatives (Brooks and Tobias, 1996). Considering the above, we explore wrongness in this paper as a matter of perspective.

Despite the previous unconsolidated opinions, it is suggested that even a "wrong" model may entail some usefulness. Castaño (1999) mentions that a model is useful if it addresses the problems it is expected to, while Jessop (2002) explains that a model is useful if it provides the groundwork for taking decisions. For Mens and Van Gorp (2006) usefulness resorts to helping system understanding and proper decision-making. Despite the various definitions on usefulness, possible uses of "wrong" models have not been explored empirically within OR, and studies on the matter are scarce. Very few exceptions are encountered. Hodges (1991), and, Hodges and Dewar (1992) reference some relevant possibilities: use to promote or communicate a selling idea, use for training support, use for storing information, exploitation of model for creating new knowledge where precision is not required, etc. Similarly, Bankes $(1993 ; 1998)$ explains in what ways exploratory and weakly predictive models can assist in decision making. We notice a gap in empirically exploring the aforementioned possible uses.

Due to the broadness of the topic, and besides the general need to address the gap on usefulness, a more focused analysis should also be considered on "wrong" models as it is lacking from literature as well. This would allow a better understanding on the ideas of accepting and using "wrong" models. In order to further focus our analysis, a specific reason of wrongness and a distinct viewpoint of examination are required. We notice that an important factor concerns a model's level of detail. Level of detail refers to a model's description of its objectives and leads to simplification as the methodological approach of reaching or applying that proper level (Innis and Rexstad, 1983). If done in extreme or if it is lacking, then we may end up with a "wrong" model (e.g. Goldberg et al., 1990). Regarding the viewpoint of examination, in OR clients/users are central to its scope, which means that acceptance of a model is very important. Since we are also investigating model usefulness, we need to consider the two notions together. We find that Landry et al. (1983) mention that there is a relation 
between model validation and usefulness but still keep the notions distinct. Indeed, usefulness is usually referenced as a separate notion from validity - and consequently credibility which is another concept related to model validation (as explained below). For example Lewandowski (1982) states that "although generally a valid model is useful this may not always be the case". In other words, accepting a model for a purpose, and, finding it useful are not necessarily one and the same. Their possible combinations can be demonstrated in Table 1:

Table 1 Juxtaposition of model acceptance and usefulness

\begin{tabular}{|c|c|c|c|}
\hline & \multicolumn{2}{|c|}{ Model usefulness (by clients) } \\
\hline & & Useful model & Not-useful model \\
\hline \multirow{2}{*}{$\begin{array}{l}\text { Model } \\
\text { acceptance } \\
\text { (by clients) }\end{array}$} & Yes & $\begin{array}{l}\text {-Accepted by clients } \\
\text {-Model identified as useful } \\
\text { by clients }\end{array}$ & $\begin{array}{l}\text {-Accepted by clients } \\
\text {-Model not identified as useful } \\
\text { by clients after all }\end{array}$ \\
\hline & No & $\begin{array}{l}\text {-Rejected by clients } \\
\text {-Model still identified as } \\
\text { useful by clients after all }\end{array}$ & $\begin{array}{l}\text {-Rejected by clients } \\
\text {-Model not identified as useful } \\
\text { by clients }\end{array}$ \\
\hline
\end{tabular}

Model acceptance here refers to clients accepting a model, initially, for their purposes. Even an accepted model may be considered "wrong" by any side due to some reason.

Table 1 shows the possible combinations between accepting a model and finding it useful. Interpreting this for "wrong" models, there are two "obvious" cases: an accepted model also considered useful, and, a rejected model not considered useful. Also, models may be rejected initially, but they may still entail some usefulness. A final - slightly contradicting but still plausible - case regards an accepted model that is not considered useful after all. We have thus expressed that usefulness may exist for a model even if it is not accepted. Its importance as a distinct idea when dealing with "wrong" models is becoming obvious. In OR, model acceptance can further be considered as a decision based on what the involved parties think of a model, that is a subjective decision following their denoted credibility. Credibility is one of the various concepts related to model validation (Tsioptsias et al., 2016). While model validation is "the process of ensuring that the model is sufficiently accurate for the purpose at hand" (Robinson, 2014), credibility is termed as the clients' belief that a model has credential value (Gass, 1983) and matches the aforementioned consideration of clients being central to the scope of OR. In other words, if a model is credible then we consider that it may be accepted. To summarise, the model level of detail is considered for exploration as a reason of wrongness under the scope of credibility to determine how acceptance and usefulness are affected.

Having elaborated on how literature views usefulness and uses of "wrong" models, as well as focusing on a specific reason of wrongness and how acceptance and usefulness are juxtaposed, we next set objectives over these topics and presents the employed methodology to address them.

\section{OBJECTIVES AND METHODOLOGY}

The above analysis suggests a gap in literature in terms of how "wrong" models can be used in practice regarding their usefulness. This section presents our approach by setting and elaborating on the objectives and our expectations, and, by introducing the research design.

\subsection{Objectives and explanations}

We consider the following. The term "wrong" is subjective and we use it for a model with at least one of its elements not considered good enough by either clients or modellers. Also, as stated in Section 1, using a "wrong" model does not equate to a modeller willingly developing or adapting a bad model.

This paper aims to examine the extent to which "wrong" models are used in practice and how modellers and clients deal with them. Based on the identified gap, we set two objectives. The first objective is to explore possible uses of "wrong" models in practice (O1). The second objective is to perform a more in-depth, focused analysis by investigating how the level of detail as a specific reason of wrongness affects model acceptance and model use (O2). Cases of "wrong" discrete-event simulation (DES) models are investigated to identify potential uses as well as to focus the analysis. 
$\mathrm{O} 1$ is a direct consequence of the gap found in literature in empirically exploring uses for "wrong" models (Section 2) and addresses the question "are 'wrong' models used in practice and how?". We would expect that if "wrong" models are used in practice, then the uses of such models could be examined, compared to literature, and, be extrapolated for use in other cases.

For O2, we specialise the investigation for level of detail as a reason of wrongness and its effect on model use and acceptance. Model acceptance is viewed as a decision following clients' perception of a model (credibility). It can result from a model's level of detail by considering a range of acceptance for the client depending on their perception as shown in Figure 1 (adapted from Robinson, 2014). Since increasing the level of detail (complexity) leads to an increase in accuracy only up to a point, we consider two points "x" and " $y$ " which deem the model as too simple ("oversimplified") or too complex ("overcomplicated"). The combination of perceived complexity and accuracy puts model acceptance "on the map" for modellers and clients. Perceived complexity is considered the subjective complexity based on someone's perception of a model. Perceived accuracy refers to the subjectively denoted accuracy leading to credibility and acceptance. The main curve shows the modeller's perception of a model, while the green and red dotted curves create a perception range for the clients (blue arrows). If their perception is "over" the modeller's (green curve) then a model deemed as oversimplified or overcomplicated may be accepted. If their perception is "below" the modeller's (red curve) then a model deemed as adequate may be rejected. The objective thus addresses the question "does the level of detail affect model acceptance and model use for 'wrong' models?". We consider that level of detail, acceptance and usefulness are interrelated and may affect each other.

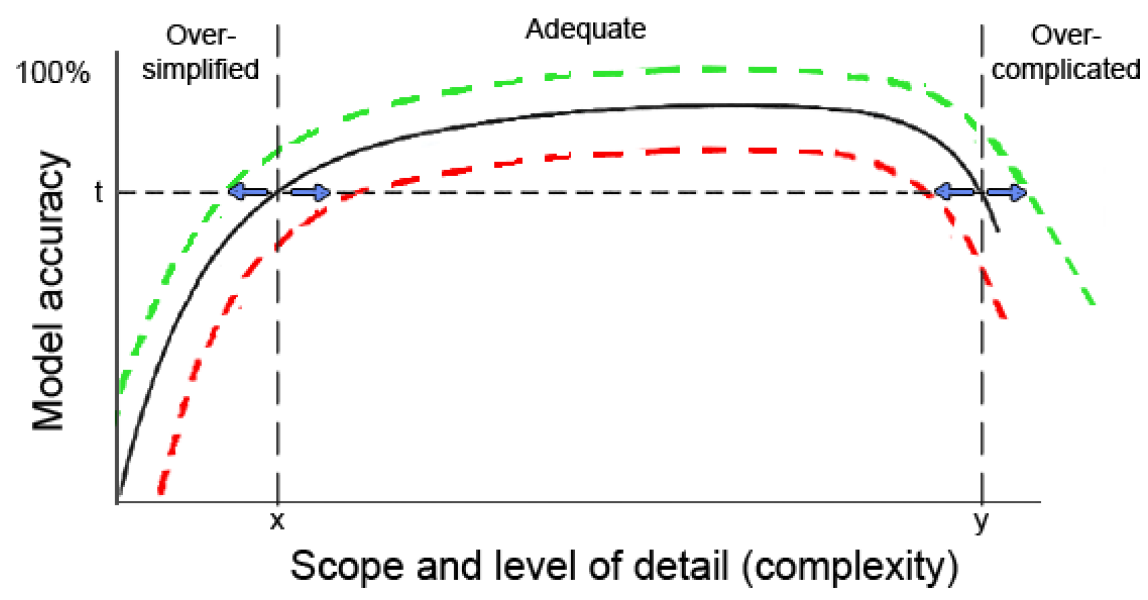

Figure 1 Adapted from Robinson (2014). Points " $x$ " and " $y$ " separate level of detail (modeller's viewpoint). The main curve shows the modeller's perception of a model. The dotted curves create a perception range for the clients leading to acceptance or rejection of models.

\subsection{Methodology and process of interviews}

To accomplish the two objectives, semi-structured interviews with DES modellers are conducted. Interviews appear as the most straightforward method in order to collect information from real-life cases compared to other qualitative methods. Their results can be categorised and compared (Gogi, 2016) in order to lead to specific deductions for the objectives and contrary to case studies and ethnography, interviews require less time and can take place remotely if required. Following, we present considerations regarding the interviews as well as information on the process followed.

The interviews required modellers to recall stories relevant to "wrong" models. Time was given in advance to the participants to recall details based on their memory. This would allow free and detailed narrative which is a requirement when conducting interviews (Brinkmann and Kvale, 2015). Another concern was the number of interviews to have enough material. It has been suggested that saturation tends to happen at around 12 interviews (Guest et al., 2006). Our case study consists of 22 interviews. Based on the amount of material collected and the outcomes (Section 4), we consider the number as adequate for our cause. Ethical guidelines are also important (Brinkmann and Kvale, 2015) and thus an ethics clearance was performed including a consent form informing every interviewee in advance. 
The process for each interview is as follow. Initially, DES modellers from different companies are contacted to invite them to participate in the study. More details are provided upon agreement, without referring to the concept of "wrong" models to avoid biasing the modellers' own understanding of the term. Each interview takes between 40 minutes and 1 hour and is done in person unless not feasible, in which case the interview is to be carried out over the phone or Skype. Once collected, all results are anonymised for confidentiality purposes. The questions were based on the objectives formulated for the interviews. We asked the interviewees to comment on each story they detailed, on problems they encountered, the clients' responses per case, and how they dealt with these situations.

Section 4 presents information on the collected material and the results deriving from the analysis.

\section{RESULTS}

The interviews took place between November 2018 and January 2019 with the modellers. We present some information on the interviews, followed by the results per objective.

\subsection{Information on sample}

A total of 22 interviews was conducted with simulation analysts that use DES as part of their job. The participating companies are from the private sector, the majority being in consulting with the exception of one, from the manufacturing sector. Modellers of different seniority contributed a total of 54 stories. Out of the 22 participants, half of them were considered experienced as 6 interviewees had relevant experience of approximately two decades within simulation and 5 more interviewees had experience of well over 20 years. A number of 6 interviewees were classified as novice, being quite new to the field (between 1 and 3 years), while the rest were classified as confident users and spanned between 4 and 8 years of work. One interviewee contributed only general input on model wrongness. All interviews were made face-to-face with the exception of two being over the phone, and one over Skype. All stories were considered relevant to "wrong" models as they presented issues related to the model or process followed, or, there was a view related to model wrongness from an involved party.

\subsection{Objective 1: Are "wrong" models used in practice and how?}

The extent to which "wrong" models are used in practice, and if so how, is tackled first.

Since all stories contained a "wrong" model, it is considered that real-life cases have to deal with such issues. It was first examined if "wrong" models were used in any manner. The interview material was analysed iteratively on whether a story's model was used by the client regardless of credibility or acceptance and how, either as direct answers to interview questions on what happened with each model or as logical deductions from each narrative. Table 2 summarises the stories as follow: A model is considered to have been "used" if there was a reference of a possible utilisation of that model in its corresponding story despite being considered "wrong". A model is considered to not have been "used" if there was a reference of no further use in its story after being considered "wrong". Any story not delivering either information for a model was separately categorised as unclear.

Table 2 Categorisation of whether "wrong" models were used

\begin{tabular}{|l|c|}
\hline Category & Number of models \\
\hline Used & $25(46.3 \%)$ \\
\hline Not used & $24(44.4 \%)$ \\
\hline Unclear & $5(9.3 \%)$ \\
\hline Total & $\mathbf{5 4}$ \\
\hline
\end{tabular}

About half of the cases were attributed to the category of using a "wrong" model in at least some manner - regardless as stated who considered it "wrong". Unclear cases regarded confidentiality issues from the modeller's clients or lack of knowledge in view of what happened next.

If a "wrong" model was thus used, then the reasons for using it and its uses were considered. Iteratively revisiting the content of the discussions, both reasons for use and specific uses of those models were grouped and categorised. Table 3 presents first the reasons for using "wrong" models. 
Table 3 Reasons for using "wrong" models

\begin{tabular}{|l|c|}
\hline Reasons & Number of models \\
\hline Further investigation & $8(32 \%)$ \\
\hline No alternative & $7(28 \%)$ \\
\hline Client happy & $7(28 \%)$ \\
\hline Third party & $3(12 \%)$ \\
\hline Total & $\mathbf{2 5}$ \\
\hline \multicolumn{2}{|l|}{ Each model is attributed to one main category of reason for being used }
\end{tabular}

Results suggest that in most stories a "wrong" model may have been used due to further exploration of the issues taking place with the modeller showing to clients that the model may still be useful regardless of wrongness. Also, a "wrong" model may have been used because there were no other present options or because changes to that model had no effect on its wrongness. A similar number of cases presents utilisation of a "wrong" model due to clients considering the model adequate for their purposes as for example being aware that the model has wrong elements but still chose to implement, or, not finding anything problematic with the model from the start. Lastly, the involvement of possible third parties had an effect on 3 cases, where a "wrong" model was inclined to be further utilised. Third parties here may refer to anyone interested in the project (e.g. a director or a client of a client) that could have affected the acceptance of a model without necessarily being involved in its development.

Next, the uses of those models are provided in Table 4. These derive from categorising the way that the models were used in the 25 cases identified to have a "wrong" model used (Table 2). The categories were created by revisiting iteratively the stories and checking for commonalities.

Table 4 Uses of "wrong" models

\begin{tabular}{|l|c|}
\hline Reasons & Number of models \\
\hline Decisions or actions & $17(68 \%)$ \\
\hline Hypothesis or scenario testing & $8(32 \%)$ \\
\hline Surface issues & $7(28 \%)$ \\
\hline Better understanding or thinking development & $3(12 \%)$ \\
\hline Promote or communicate selling & $2(8 \%)$ \\
\hline Store or monitoring & $1(4 \%)$ \\
\hline
\end{tabular}

A model may be attributed to more than one categories of uses

The categories suggest that a "wrong" model may have been used most often for helping in decisionmaking, exploring options on actions. Training support is included in this category. The second most often use refers to helping the clients experiment on parameters when outcomes are not necessarily required to be numerically perfect, followed by models used to highlight problems that involve the clients or their collaborators. Next, a model may have been used because it allowed insights to the simulated reality. Lastly, some cases were found where a "wrong" model was used to either promote a selling idea or for storing information. As a final note, if a model was not used, two main reasons were found: the models were either not used at all ( 6 cases - 25\%), or, they were changed into an "adequate" model and then used thus not being considered "wrong" any more (18 cases - 75\%).

Concluding O1, we have found that "wrong" models are indeed encountered and also used in practice quite often. Reasons and uses were identified and categorised. Next, we focus our analysis.

\subsection{Objective 2: Does the level of detail affect model acceptance and use?}

Having explored whether "wrong" models are used in practice as well as their uses, we now focus on level of detail and its effect on model acceptance and use. This specification will help us understand better how model acceptance and use may interact based on an exemplified reason of wrongness.

A total of 13 out of the 54 stories highlighted issues related to level of detail. These could have derived from modellers, clients or both. Models considered simpler than expected or to be missing elements were denoted as "oversimplified" (OsM), and, models considered too complex or having 
more elements than required were denoted as "overcomplicated" (OcM). Table 5 presents the number of models viewed as "wrong" by modellers and clients due to their level of detail.

Table 5 Number of "wrong" models due to level of detail

\begin{tabular}{|l|c|c|c|}
\hline & Modellers & Clients & Unique "wrong" models \\
\hline OsM & 4 & 4 & 7 \\
\hline OcM & 8 & 4 & 8 \\
\hline "Wrong" models per side & $\mathbf{1 2}$ & $\mathbf{8}$ & $\mathbf{1 3}$ \\
\hline
\end{tabular}

OsM = Oversimplified models, OcM = Overcomplicated models

In our sample, the modellers tend to denote wrongness due to level of detail more often than the clients, with 12 cases compared to 8 . Additionally, the modellers here reference more often issues with OsM than OcM, with 8 cases compared to 4 . The clients' concerns in 8 stories are equally split between OsM and OcM. In 5 out of these 8 stories the opinions coincide and in 2 stories the opinions are opposite to the modellers while in 1 story only the clients denoted issues. In total, 7 stories referenced an OsM and 8 an OcM, repeating counting for the 2 models with opposite views.

Comparing the above with model acceptance, we get the following correspondence in Table 6.

Table 6 Number of "wrong" models and model acceptance

\begin{tabular}{|l|l|c|c|c|}
\hline & & Modellers & Clients & Unique models \\
\hline \multirow{3}{*}{ Accepted by clients } & OsM & 4 & 2 & 5 \\
\cline { 2 - 5 } & OcM & 2 & 1 & 2 \\
\cline { 2 - 5 } & Total & $\mathbf{6}$ & $\mathbf{3}$ & $\mathbf{7}$ \\
\hline \multirow{3}{*}{ Not accepted by clients } & OsM & 0 & 2 & 2 \\
\cline { 2 - 5 } & OcM & 6 & 3 & 6 \\
\cline { 2 - 5 } & Total & $\mathbf{6}$ & $\mathbf{5}$ & $\mathbf{6}$ \\
\hline
\end{tabular}

Two models are viewed differently by modellers/clients and are repeated in counting (in red).

OsM = Oversimplified models, OcM = Overcomplicated models

Model acceptance was deducted based on the interviewees' views of their clients' credibility. Out of the 13 models, 7 were accepted and 6 rejected. From Tables 5 and 6, we notice that 6 out of the 12 models with issues denoted by the modellers were accepted. On the contrary, only 3 out of the 8 models with issues denoted by the clients were accepted. This is expected, as the clients may have a stronger opinion on wrongness and acceptance. We highlight that 5 out of the 7 OsM were accepted, contrary to only 2 out of the 8 OcM. This could mean that OsM can be more easily apprehended.

Regardless if a model was initially accepted, we also check whether that model was further used. Though we do not refer here to the reasons and ways of using or not using a model, we consider that an accepted model may have ended up not being used, while a rejected model may have still been found useful (see Table 1). By iterating the material, in view of usefulness we notice that: Out of the 7 accepted "wrong" models, 5 were actually used which means that 2 models despite initial acceptance were not further utilised. Regarding these 2 models not used, 1 of them was considered as oversimplified by the modeller but adequate by the clients while the other one was considered adequate by the modeller but overcomplicated by the clients. Out of the 6 rejected "wrong" models, 5 were not used which means that 1 model despite initial rejection was further utilised. That model was considered overcomplicated by the modeller and the clients but they still found some use in it. It is noted that the 2 models with contrasting opinions on their complexity were neither accepted nor used.

Table 7 Comparing acceptance and use of "wrong" models due to level of detail

\begin{tabular}{|l|c|c|c|}
\hline & Used & Not used & Total ("wrong" models) \\
\hline Accepted by clients & 5 & 2 & 7 \\
\hline Not accepted by clients & 1 & 5 & 6 \\
\hline Total ("wrong" models) & $\mathbf{6}$ & $\mathbf{7}$ & $\mathbf{1 3}$ \\
\hline
\end{tabular}


Table 7 summarises the discussed combinatory outcomes of acceptance and usefulness. Each of these 13 models could be depicted on Figure 1 based on level of detail and acceptance.

Consolidating Tables 5-7, we notice that a model denoted by the clients as "wrong" due to its level of detail might be accepted but not necessarily used and vice versa. For example, an accepted OsM denoted as such by the clients is not used after all, while 1 of the 2 used models that was denoted as OcM by the clients was not initially accepted. Furthermore, there is an equal split for the models where the modeller considered level of detail as an issue in view of their acceptance as well as use.

Table 7 is an empirical evaluation of Table 1 and corroborates the claims made in view of acceptance and usefulness. Our expectation that level of detail, acceptance and usefulness affect each other cannot be supported or rejected quantitatively as the sample is very small, but from a qualitative point of view we could state the following: an accepted model has a higher chance of being used, and, the level of detail as an issue for a model does not seem to affect acceptance as often when deriving from the modellers' side but it is taken into better consideration when deriving from the clients' side. These deductions suggest that models may be denoted as "wrong" by a side but still be accepted and/or used while their acceptance may not necessarily coincide with the usefulness they may provide to their clients after all. This focused analysis could be repeated for other reasons of wrongness.

To summarise, our in-depth investigation on one specific reason of wrongness and its effect on acceptance and usefulness offered some interesting inputs. We found that opinions on level of detail do not always coincide and may even differ, with modellers addressing level of detail more often as a problem for models. Also, accepting a model seems more related to cases where the clients did not have a negative opinion of the model since clients may have a stronger opinion on model wrongness. Yet, this may still change and they may utilise an initially rejected model. Lastly, oversimplified models may be accepted more frequently than overcomplicated ones. The next section summarises and discusses the work of the paper, alongside limitations and future expansions.

\section{DISCUSSION, LIMITATIONS AND FUTURE WORK}

This paper is the first to address in practice the possible usefulness that "wrong" models may still entail within OR. After presenting how literature regards model usefulness and acceptance, a research gap was encountered. Two objectives were set to address the gap: whether and how "wrong" models are used when encountered in practice, and, a focused investigation combining a specific reason of wrongness and its effect on model acceptance and model use. Interviews with DES modellers to explore the objectives were conducted, discussing DES modelling stories. Our aim was to provide a better understanding on "wrong" models by exploring the extent and way to which they are used in practice. The most relevant findings are discussed here followed by study limitations and future work.

The possible usefulness of "wrong" models is lacking in literature. We showed how model acceptance can be juxtaposed with usefulness, suggesting that the two may not coincide. Still, and despite the plethora of reasons for model wrongness, the possible uses of models considered "wrong" had not been addressed in practice.

The examination of the first objective showed that "wrong" models are indeed encountered and utilised in practice in at least some manner for almost half of the sample's examined cases. Reasons for using "wrong" models were identified and categorised. The most often cases included models used for helping with decision support or hypothesis testing, and the reasons for using them were most often due to lack of alternatives or because clients were happy with a specific model regardless if they found it "wrong", or, due to models being proven to have some value by the modellers to the clients. It is thus easily suggested that views on wrongness may differ or even collide. A "wrong" model for a modeller may seem perfectly fine for a client. Or, vice versa, a client may dismiss a model that is found to be adequate by its modeller. This finding, alongside the possible uses of "wrong" models, can be utilised when the two sides - modellers and clients - derive to unconsolidated opinions on a model, i.e. even if the model is deemed as "wrong" there may be usefulness and specific uses to it. The uses of "wrong" models explain as well the fact that the subjectively denoted idea of a created model being unfit for a cause can still find fruitful derivatives for a client.

The analysis on the stories for issues on their models' level of detail for O2, offered some further understanding on how that specific reason of wrongness affects model acceptance as a decision 
following credibility, and, model use. It was suggested from the sample that modellers denote wrongness due to level of detail more often than clients but opinions do not always coincide and may even be opposite. It was inclined that clients considering a model "wrong" may still accept it but not necessarily use it and vice versa. Additionally, clients have a stronger effect on acceptance or rejection of a project than modellers (as expected), especially for oversimplified compared to overcomplicated models. Yet, their opinions on using or not a model may be altered by the modellers. These deductions lead to the idea that accepting and using a model does not necessarily coincide when level of detail is the main attributed reason of wrongness, nor does the opinions between the modeller and the clients in view of the assigned level of detail of a model (too much or not enough).

The contributions of our work can be used as empirical evidence and ideas to help support the interaction between clients and modellers in case of disagreement on model complexity/simplicity as well as the utilisation of such a model regardless of the initial acceptance or rejection. Indeed, a model may be rejected by a client but if the modeller can prove its further usefulness, it may still apply to some extent as an additional tool for decision-making or some other function.

A number of limitations apply to this study. The stories were reviewed from the modellers' perspectives, thus an extension to clients or validation of results with their help would allow more concrete deductions. The analysis considered only one reason of wrongness under the scope of credibility, while the sample was very limited with only 13 models referencing this reason. Possible interrelated reasons between issues leading to model wrongness and their effect on accepting or using a model could apply, which has not been tackled here. The interaction with the clients' and modellers' decisions could be further explored. Lastly, all of the above analysis is subject to interpretation bias.

The above pave the way towards a further examination of how "wrong" models may be of use to both clients and modellers through the idea of learning, since using a "wrong" model may offer specific benefits. In future work, the authors aim to further analyse the interviews, under the scope of learning, and to identify how different stakeholders interact with "wrong" models when encountering them. As a result we aim to develop a framework of interaction between the stakeholders involved in a simulation study to suggest possible courses of action when "wrong" models are encountered.

\section{ACKNOWLEDGMENTS}

The authors acknowledge the support of SIMUL8 Corporation for offering a travel grant to support research activities of the $\mathrm{PhD}$ project that this paper is part of. The authors would also like to thank all the simulation modellers and their companies for taking part in the interviews presented in this paper.

\section{REFERENCES}

Balci, O., Nance, R. E., Arthur, J. D. and Ormsby, W. F. (2002). Expanding our horizons in verification, validation, and accreditation research and practice. Proceedings of the Winter Simulation Conference, 1(2000), pp 653-663.

Bankes, S. (1993). Exploratory Modeling for Policy Analysis. Operations Research, 41(3), 435-449.

Bankes, S. (1998). Policy Analysis for Complex and Uncertain Systems Through Computational Experiments. In IEEE Aerospace Conference, pp 9-21, vol.1.

Brooks, R.J. and Tobias, A.M. (1996). Choosing the best model: Level of detail, complexity, and model performance. Mathematical and Computer Modelling, 24(4), pp 1-14.

Brinkmann, S. and Kvale, S. (2015) Interviews: Learning the Craft of Qualitative Research Interviewing. 3rd Edition, Sage Publications, Thousand Oaks, CA.

Box, G. E. P. and Draper, N. R., (1987) Empirical Model-Building and Response Surfaces, John Wiley \& Sons.

Castaño, Y. Á. (1999). Dynamic Behavior of NPD. Proceedings of the 17th International Conference of the System Dynamics Society and 5th Australian \& New Zealand Systems Conference, 10.

Eskinasi, M. and Fokkema, E. (2006). Lessons learned from unsuccessful modelling interventions. Systems Research and Behavioral Science, 23, pp 483-492.

Gass, S. I. (1983). Decision-Aiding Models: Validation, Assessment, and Related Issues for Policy Analysis. Operations Research, 31(4), pp 603-631. 
Gogi, A. (2016). Insight generation in simulation studies : an empirical exploration.

Groesser, S. and Schwaninger, M. (2012). Contributions to model validation: hierarchy, process, and cessation. System Dynamics Review, 28(2), pp 157-181.

Guest, G., Bunce, A. and Johnson, L. (2006). How Many Interviews Are Enough? Field Methods, 18(1), pp 59-82.

Hodges, J. and Dewar, J. (1992). Is it you or your model talking? A framework for model validation. Retrieved from http://www.rand.org/content/dam/rand/pubs/reports/2006/R4114.pdf

Hodges, J. S. (1991). Six (Or So) Things You Can Do with a Bad Model. Operations Research, 39(3), pp 355-365.

Innis, G. and Rexstad, E. (1983). Simulation model simplification techniques. Simulation, 41, 7-15.

Jessop, A. (2002). Exploring structure: A blockmodel approach. Civil Engineering and Environmental Systems, 19(4), pp 263-284.

Landry, M., Malouin, J.-L. and Oral, M. (1983). Model validation in operations research. European Journal of Operational Research, 14(3), pp 207-220.

Lewandowski, A. (1982). Issues in model validation. International Institute for Applied Systems Analysis, RR-82-37(March), pp 1-26.

Mens, T. and Van Gorp, P. (2006). A taxonomy of model transformation. Electronic Notes in Theoretical Computer Science, 152(1-2), pp 125-142.

Robinson, S. (2014). Simulation - The Practice of Model Development and Use, 2nd edition. Macmillan education, Palgrave.

Sargent, R.G. (2012). Verification and validation of simulation models. Journal of Simulation, 7(1), pp 12-24.

Tsioptsias, N., Tako, A. and Robinson, S. (2016). Model Validation and Testing in Simulation: a Literature Review. 5th Student Conference on Operational Research, 6:1-6:11.

Tsioptsias, N., Tako, A. and Robinson, S. (2018). Can we learn from wrong simulation models? A preliminary experimental study on user learning. Proceedings of the Operational Research Society Simulation Workshop 2018 (SW18).

Vennix, J. A. M. (1999). Group model-building: tackling messy problems. System Dynamics Review, 15(4), pp 379-401.

\section{AUTHOR BIOGRAPHIES}

NAOUM TSIOPTSIAS is a Doctoral Researcher at Loughborough University in Operational Research. He received a Bachelor on Finance \& Accounting from the University of Macedonia (Thessaloniki) and an MSc on Informatics \& Management from the Aristotle University of Thessaloniki. His current research concerns "wrong" DES models uses, while previous interests regarded data mining on financial crises. Ha has also worked as an analyst for an HR company.

ANTUELA TAKO is a Reader in Operational Research at the School of Business and Economics, Loughborough University. She holds a PhD in Simulation and an MSc in Management Science and Operational Research from the University of Warwick. Her research interests include the comparison of simulation approaches, facilitated and participative simulation modelling, conceptual modelling and health care modelling. She is an Associate Fellow of the Operational Research Society (AFORS), Associate Editor of the Journal of the Operational Research Society and the Journal of Simulation.

STEWART ROBINSON is Dean and Professor of Management Science at Loughborough University, School of Business and Economics. Previously employed in simulation consultancy, he supported the use of simulation in companies throughout Europe and the rest of the world. He is author/co-author of six books on simulation. His research focuses on the practice of simulation model development and use. Key areas of interest are conceptual modelling, model validation, output analysis and alternative simulation methods (discrete-event, system dynamics and agent based). Professor Robinson is co-founder of the Journal of Simulation and the UK Simulation Workshop conference series. He was President of the Operational Research Society (2014-2015). Home page: www.stewartrobinson.co.uk. 